Casos Clínicos

Arch. Esp. Urol., 60, 1 (84-85), 2007

\section{INSUFICIENCIA RENAL AGUDA SECUNDARIA AL SÍNDROME DE RESECCIÓN TRANSURETRAL DE PRÓSTATA.}

Faissal Tarrass, Meryem Benjelloun, Khadija Hachim, Ghislaine Medkouri, Mohamed Gharbi Benghanem y Benyounes Ramdani.

Servicio de Nefrología y Diálisis. Hospital Universitario Ibn Rochd. Casablanca. Marruecos.

Resumen.- OBJETIVO: Las complicaciones de la resección transuretral de próstata (RTUP) relacionadas con la hipotonía y la hipervolemia fueron descritas bien en la literatura. La insuficiencia renal aguda, es una forma de presentación clínica que puede surgir en el curso de la resección transuretral de próstata, y que fue poco discutida.

MÉTODOS. Presentamos un caso de insuficiencia renal aguda oligúrica como importante complicación después de la RTUP, con una discusión de un posible mecanismo para el desorden.

RESULTADOS. En nuestro paciente, el mecanismo por el cual se produjo el fallo renal no está claro, aunque posiblemente fuese debido a una hemólisis. Otros factores etiológicos, tales como la alteración hemodinámica, hipotensión, o rabdomiolisis fueron implicadas en base a los datos analíticos.

CONCLUSIONES. Creemos que una evaluación detallada en el transcurso de una RTUP, pueden ayudar a minimizar la incidencia y prevenir esta anormalidad.

Palabras clave: Resección transuretral. Prostatectomía. Hemodilución. Hiponatremia. Insuficiencia renal aguda.

Faissal Tarrass

Salama 3, Gr 6, B, No 21

20450 Casablanca. (Marruecos).

faissal76@hotmail.com

Trabajo recibido: 29 de mayo 2006
Summary.- OBJECTIVE: Complications of transurethral resection of the prostate (TURP) related to hypotonicity and hypervolemia were well described in the literature. However acute renal failure, kown to be a clinical presentation of some TURP syndromes was less discussed.

METHODS. We report a case of oliguric acute renal failure as a major complication after TURP, with a discussion of a possible mechanism for the disorder.

RESULTS. The mechanism by which renal failure developed in our patient is not entirely clear, but most likely is due to hemolysis. Other factors such as hemodynamic alterations, hypotension and rabdomyolisis are also suspected based on analysed data.

CONCLUSION. We believe that a detailed evaluation before and after TURP will allow to reduce the incidence of this abnormality and to prevent its occurrence.

Keywords: Transurethral resection. Prostatectomy. Hemodilution. Hyponatremia. Acute renal failure.

\section{INTRODUCCIÓN}

A pesar del desarrollo de técnicas no invasivas para el tratamiento de la hiperplasia benigna prostática, el tratamiento quirúrgico que, en muchas ocasiones es la resección transuretral (RTU), constituye la opción terapéutica más eficaz para aquellos pacientes que no responden favorablemente a la farmacoterapia (1). La absorción de líquidos hipotónicos utilizados para la irrigación vesical durante el procedimiento pueden causar alteraciones hemodinámicas y del sistema nervioso central que, en su conjunto o por separado, se conocen como "síndrome de reabsorción o de resección transuretral" (síndrome RTU) $(1,2)$.

La insuficiencia renal aguda, es una de las complicaciones que pueden surgir en el curso de la RTU de próstata $(3,4)$, y está escasamente publicada en la literatura. Presentamos el caso de un paciente que desarrolló insuficiencia renal aguda secundaria e irrigación con agua estéril en el seno de RTU de próstata.

\section{CASO CLÍNICO}

Varón de 65 años, $70 \mathrm{~kg}$ de peso sin antecedentes de interés que acudió a nuestro hospital por RTU de próstata. Desde hace un año y medio presenta sintomatología de síndrome prostático. Estudiado por el Servicio de Urología, se le diagnostica una hipertrofia benigna de próstata. El examen preoperatorio no presentaba alteraciones (electrocardiograma, hemograma y bioquímica normales). Se programa para RTU. No se realizo premedicación. La anestesia intrarraquídea se realiza con el paciente en sedestación. Antes de la inducción anestésica se monitorizo un electrocardiograma continuo (ECG), una presión arterial (PA) no invasiva y la 
SpO2. La intervención tuvo duración de 60 minutos y transcurrio sin incidencias. Durante la misma se administraron 3,5 litros de agua estéril (hipotónica) como liquido de irrigación, contabilizandose un balance negativo de $1500 \mathrm{ml}$. En la sala de despertar presento vómitos e hipotensión $(100 / 60 \mathrm{mmHg})$. No se objetivaron alteraciones neurológicas, ni cardiovasculares, ni edema pulmonar. ECG y Rx torax sin alteraciones. En la bioquímica sanguínea destacaba: sodio : $122 \mathrm{mEq} / \mathrm{L}$, potasio : $5,9 \mathrm{mmol} / \mathrm{L}$, osmolaridad : $256 \mathrm{mOsmol} / \mathrm{kg}$, urea : 1,5 $\mathrm{g} / \mathrm{L}$, creatinina (cr.s) : $38 \mathrm{mg} / \mathrm{L}$, calcio : $80 \mathrm{mg} / \mathrm{L}$, proteínas totales : $51 \mathrm{~g} / \mathrm{L}$, albúmina: $28 \mathrm{~g} / \mathrm{L}, \mathrm{CPK}: 225$ U/L, LDH : $416 \mathrm{U} / \mathrm{L}, \mathrm{GOT}: 48 \mathrm{U} / \mathrm{L}, \mathrm{GPT}: 97 \mathrm{U} / \mathrm{L}$, haptoglobina : $43.8 \mathrm{mg} / \mathrm{dL}$ (rango normal $50-220 \mathrm{mg} / \mathrm{dL}$ ), las pruebas de coagulación salieron normales, $\mathrm{Hb}: 10$ $\mathrm{g} / \mathrm{dl}, \mathrm{HCT}: 28.8 \%$, leucocitos : $6400 / \mathrm{mm}^{3}$, plaquetas : 220.000/mm ${ }^{3}$. Gasometría: $\mathrm{pH}: 7,30 ; \mathrm{PCO}_{2}: 36$ $\mathrm{mmHg}$; PO2 : $72 \mathrm{mmHg}$. Se procede a su ingreso en la unidad de Reanimación.

Tras la estabilización clínica con normalización de la tensión arterial, se pudo prescindir de las drogas vasopresoras, se administraron Furosemida IV y suero salino hipertónico al 3\% para minimizar la sobrecarga de volumen. A pesar de las medidas aplicadas persistia con insuficiencia renal aguda oligúrica durante 15 días, llegando a cifras máximas de urea de $2,70 \mathrm{~g} / \mathrm{L}$ y de creatinina de $72 \mathrm{mg} / \mathrm{L}$, precisando de 04 sesiones de hemodiálisis. A las 3 semanas, reinició la diuresis entrando en fase poliúrica, recuperandose así progresivamente la función renal, siendo la Cr.s. al alta de $14 \mathrm{mg} / \mathrm{L}$. A los 4 meses el paciente permanece normotenso, con cifras de Cr.s. $10 \mathrm{mg} / \mathrm{L}$ y un Ccr. $76 \mathrm{ml} /$ minuto.

\section{DISCUSIÓN}

El síndrome RTU, viene definido por una serie de síntomas clínicos del sistema nervioso central y cardiovasculares (inestabilidad hemodinámica, sedación, alteraciones visuales, coma...etc), presentes en mayor o menor grado en un 10 a $15 \%$ de las RTU, con una tasa de mortalidad de $0.2-0.8 \%(2)$. Durante la resección los grandes senos venosos estan abiertos y el paso del líquido de irrigación utilizado para distender la vejiga, visualizaría y lavaria los coágulos y desechos del tejido prostático resecado, induciendo un síndrome de reabsorción $(1,2)$. El síndrome RTU habitualmente se desarrolla durante la intervención o en el post-operatorio inmediato. Los síntomas y signos iniciales incluyen bradicardia, hipotensión y algunas alteraciones neurológicas (náuseas, vómitos, confusión, inquietud, etc.). Si el síndrome progresa, se pueden originar otras alteraciones cardiovasculares, neurológicas, renales y hematológicas más severas, llegando incluso al coma y la muerte $(1,2)$.

La patogenia del síndrome es compleja. La hiponatremia refleja una hiperhi-dratación celular, que asociada a la hipoosmolaridad conlleva las siguientes complicaciones hemodinámicas y metabólicas $(5,6)$. La alteración renal se puede producir por varios mecanismos: insuficiencia renal prerrenal en pacientes muy graves, con inestabilidad hemodinámica e hipotensión (4), necrosis tubular aguda (4), hemólisis $(3,7)$, rabdomiolisis $(3,8)$. En nuestro paciente, la precipitación de la hemoglobina en el túbulo renal produce un fallo renal agudo y una hiperpotasemia. El mecanismo por el cual se produjo el fallo renal no está claro, aunque posiblemente fuese debido a una hemólisis. Otros factores etiológicos, tales como la alteración hemodinámica, hipotensión, o rabdomiolisis fueron implicadas en base a los datos analíticos.

En caso de instauracion de la insuficiencia renal, la diuresis debe ser inducida cuando las medidas de soporte hayan sido aplicadas y la presión arterial sistólica sea al menos de $90-100 \mathrm{mmHg}$., para permitir una adecuada perfusión renal. El manitol puede ser más efectivo que los diuréticos de asa, ya que actúa independientemente de los niveles de $\mathrm{Na}^{+}$al inducir una menor excreción renal del mismo. Ante el fracaso de los diuréticos, se puede volver a intentar su administración, después de aportar suero salino hipertónico $(1,2)$. Cuando todas las medidas terapéuticas fracasan se ha propuesto el uso de diálisis peritoneal y/o técnicas de hemodiálisis. Nuestro paciente precisó cuatro sesiones, comenzando posteriormente con poliuria y recuperación total de la función renal.

En resumen, aunque el mecanismo por el cual se produce la insuficiencia renal aguda en los pacientes es multifactorial. La recuperación completa de la función renal es la norma.

\section{BIBLIOGRAFÍA Y LECTURAS RECOMENDADAS ( ${ }^{*}$ lectura de interés $y$ ** lectura fundamental)}

*1. CLEMENTE RAMOS, L.M.; RAMASCO RUEDA, F.; PLATAS SANCHO, A. y cols.: "Síndrome de reabsorción post-resección transuretral (R.T.U.) de próstata: revisión de aspectos fisiopatológicos, diagnósticos y terapéuticos". Actas Urol Esp; 25: 14. 2001.

GRAVENSTEIN, D.: "Transurethral resection of the

2. prostate syndrome: a review of pathophysiology and management". Anesth Analg; 84: 438. 1007.

HUNG, C.L.; WU, C.J.; YANG, S. y cols.: “Acute re-

*3. nal failure directly caused by hemolysis associated with transurethral resection of the prostate". Urology; 59: 137. 2002.

BILEN, C.Y.; SAHIN, A.; OZEN, H. y cols.: "Nonoli-

$* * 4$. guric renal failure after transurethral resection of prostate". J. Endourol.; 13: 751. 1999.

GHANEM, A.N.; WARD, J.P.: "Osmotic and metabolic

*5. sequelae of volumetric overload in relation to the TUR syndrome". Br J. Urol.; 66: 71. 1990.

DIXON, B.; ERNEST, D.: "Hyponatremia in the tran-

6. surethral resection of the prostate syndrome". Anaesth Intensive Care; 24: 102. 1996.

CREEVY, C.D.; WEBB, E.A.: "A fatal hemolytic reac-

7. tion following transurethral resection of the prostate gland". Surgery; 21: 56. 1947.

MITNICK, P.D.; BELL, S.: "Rhabdomyolysis associa-

8. ted with severe hyponatremia after prostatic surgery". Am. J. Kidney Dis; 16: 73. 1990. 